\title{
Curve Path Detection in Autonomous Vehicle using Deep Learning
}

\author{
Kodi Balasriram, Manimozhi M \\ School of Electrical and Electronics Engineering, Vellore Institute of Technology \\ Vellore, Tamilnadu-632014, India \\ E-mail: balasriram.kodi@gmail.com, mmanimozhi@vit.ac.in.
}

\begin{abstract}
In the field of autonomous vehicles, lane detection and control play an important role. In autonomous driving the vehicle has to follow the path to avoid the collision. A deep learning technique is used to detect the curved path in autonomous vehicles. In this paper a customized lane detection algorithm was implemented to detect the curvature of the lane. A ground truth labelling tool box for deep learning is used to detect the curved path in autonomous vehicle. By mapping point to point in each frame $80-90 \%$ computing efficiency and accuracy is achieved in detecting path.
\end{abstract}

Keywords: autonomous vehicles, lane detection, curve path detection, convolutional neural networks, deep learning

\section{INTRODUCTION:}

The development of automation and electrification, autonomous robotics and vehicles has a wide range of use in the space science and self-driving cars. An important part in the autonomous vehicles is navigation system. In the past decades using vision based systems guidance system was become more popular. By taking the tire and road interaction from the vehicles automatically the terrain has to be classified these type of technique was using in the rovers and path finder now it came to the automobiles [1].

The natural frames are analysed in RGB vector space to research the feasibility of the curve path detection in automobiles. The pixels of the segmented images as been pre-processed and analysed from the curved roads. In order to obtain the curvature of the curved road least-squares curve fitting method has been used [2]. B-snake based lane detection technique and tracking algorithm was developed to identify the curve path without any camera parameters. A minimum mean square error (MMSE) was proposed to determine the control and curvature of the path [3]. Detecting the obstacle and sign board distance measurement was measured from optical sensors [4] .

A GPS based urban navigation system, based on the context awareness technique was used to detect the vehicles and fallow the lane boundaries [5]. Lane quest is he technique or the system that uses the low-energy inertial sensors available in the smart phones to provide the accurate vehicle lane detection and from the smart phone sensors surrounding environment is detected for the vehicles and the lanes [6]. To identify the lane boundaries more accurate between the entry or exit and the lane change from one lane to another lane with colliding the other vehicles an radar based tracking identification technique developed by the goodness of fit (GOF) concept to identify the position of the vehicles in the curved paths [7]. Image processing and steering control technique was used in the lane and vehicle detection by Kevin Mc Fall [8]. A special radar named all-weather automotive millimetre wave (MMW) radar was used to describe the frequency modulation of a coplanar wave radar design is capable for identifying the obstacles in the filed view by Mark E. Russell, Arthur Crain, Anthony Curran, Richard A. Campbell, Clifford A. Drubin, and William F. Miccioli [9]. A monocular vision based lane detection is developed by using on board lane detection system 
in intelligent vehciles by Xiaodong Miao, Shuming Li, Huan Shen [10]. Radar reflectors system was used in the lane boundaries to detect the lane and the vehicle automation by Alexey Voronov, johan Hulte n, Johan Wedlin, CristoferEnglund, Viktoria Swedish [11]. An automatically intelligent trajectory generator was developed and used for the lane and curve path detection by Joshue Perez Rastelli and ray Lattarulo and Fawzi Nashashibi [12]. An Predictive virtual lane algorithm was developed to mark the lane boundaries using the vision by Young Seop, Wonhee Kim, Seung-Hi Lee, and Chung Choo Chung [13]. An GPS error through filter for enhanced performance of waypoint following guidance methods has been developed and applied in the lane boundary detection by Byung-Hyun Lee, sung-Hyuck Im, Moon-Beom Heo and Gyu-In Jee [14]. Evaluation matrices and lane estimation and accuracy algorithm was used in the calculating the accuracy of the lane boundary detection by Ravi Kumar Satzoda, Mohan M. Trivedi [15]. A new lane detection algorithm was used to detect the lanes in the road using computer vision by Quoc-Bao Truong and ByungRyoung Lee [16].

\section{PATH RECOGINITION:}

\subsection{Video Processing :}

For curve path detection in autonomous vehicles using deep learning in MATLAB a particular sequence has to be followed as shown in the figure-1. By suing the video acquisition / computer vision tool box the live video has to be recorded. The recorded video has been imported to the given automated driving system tool box used in the matlab for vehicle and lane detection. From that tool box the video has been imported to groundtruth labler app in which the lane detection algorithm was applied in the each and every frame. In each frame region of interest has to be defined for the lane detection and curve path detection.

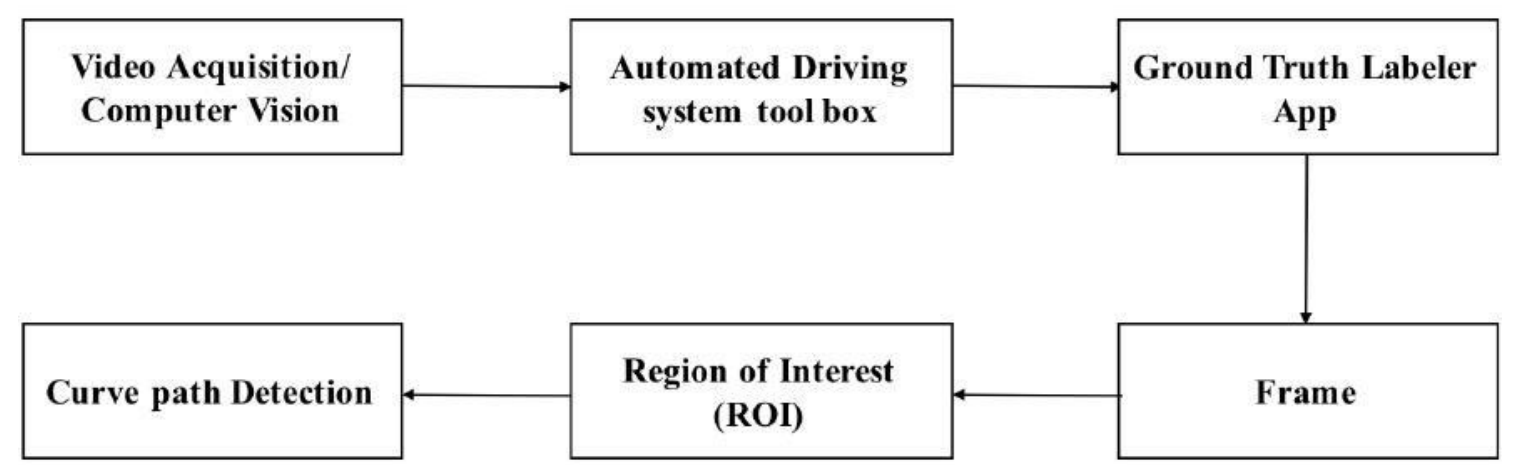

Figure: 1 Block diagram form curve path detection using deep learning

\subsection{Flow chart for Automatic Curve Path detection Algorithm:}

The Figure-2 represents the flow chart of the automatic curve path detection algorithm using deep learning technique. In which each frame in the video is processed for every $0.04 \mathrm{sec}$ and path is mapped automatically. To improve the video clarity or picture clarity image enhancement take place for given time limit. After the image enhancement edge detection take place in the image for the lane detection. Feature extraction takes place in the picture and road line or lane boundary is constructed in the video/image. To detect the curve path in video/picture curvature estimation is required for this vector lane concept is used in which curvature is estimated in the lane detection. In this case, a non-uniform B-spline interpolation is constructed to detect the left and right boundaries in the video/Image. The same execution process is continuous for all the frames till end. 


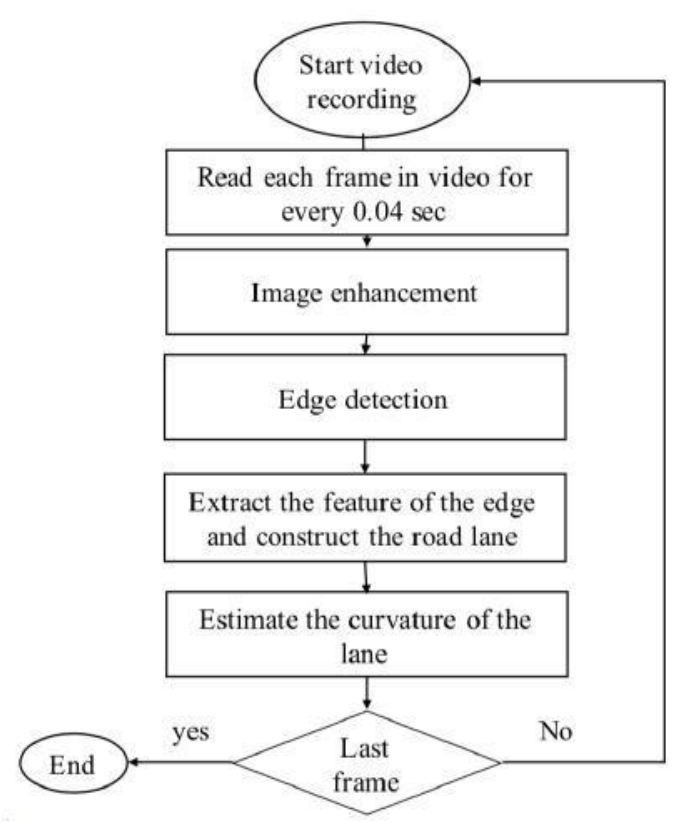

Figure:2 Flow chart Automatic Curve path detection Algorithm

\subsection{Developing and Execution Process of Automatic Curve path detection Algorithm}

\subsubsection{Creating Automation Algorithm for ground truth labelling:}

create a folder named automation with the label +driving or +automation in the matlab default folder path. Define a class that inherits from the automation algorithm class, at the matlab command prompt, enter ground truth labeller to open the app . then click select algorithm > add algorithm> create new algorithm to open the driving . automation . automation algorithm class template . Save the file to package folder to use the custom algorithm. To start the custom algorithm refresh the algorithm list in the algorithm folder in app and select the newly created lane detection algorithm.

2.3.2 Execution Process of Curve path detection algorithm:

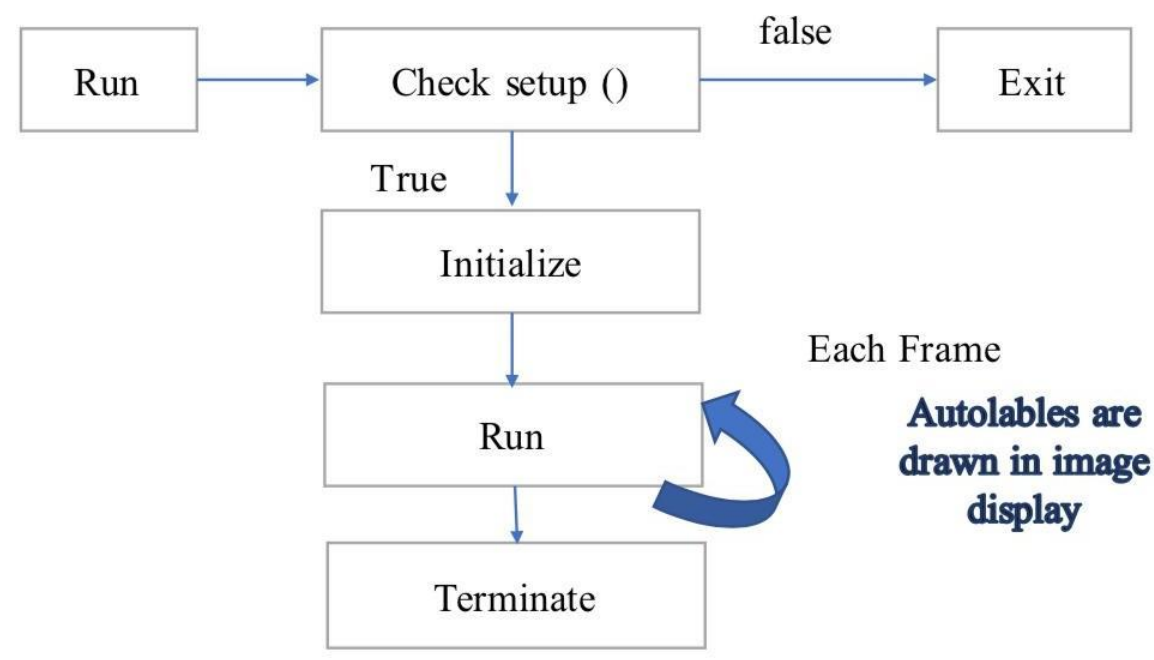

Figure:3 Execution process of curve path detection algorithm 
To execute the curve path detection algorithm, the first step to run the setup file and it checks the setup if it is true it continues the execution process or else it will exit. If set up it run perfectly without any errors it initializes the parameters based on the each frame in the video. If there is no video running the algorithm is terminated.

\section{RESULTS AND DISCUSSION}

To detect the curve path using the computer vision as shown in the figure- 4 first we have define the region of interest (ROI) where the lane boundaries are included in that. By defining the region of interest we detect the lane boundaries within that area so that possibility errors is minimum.

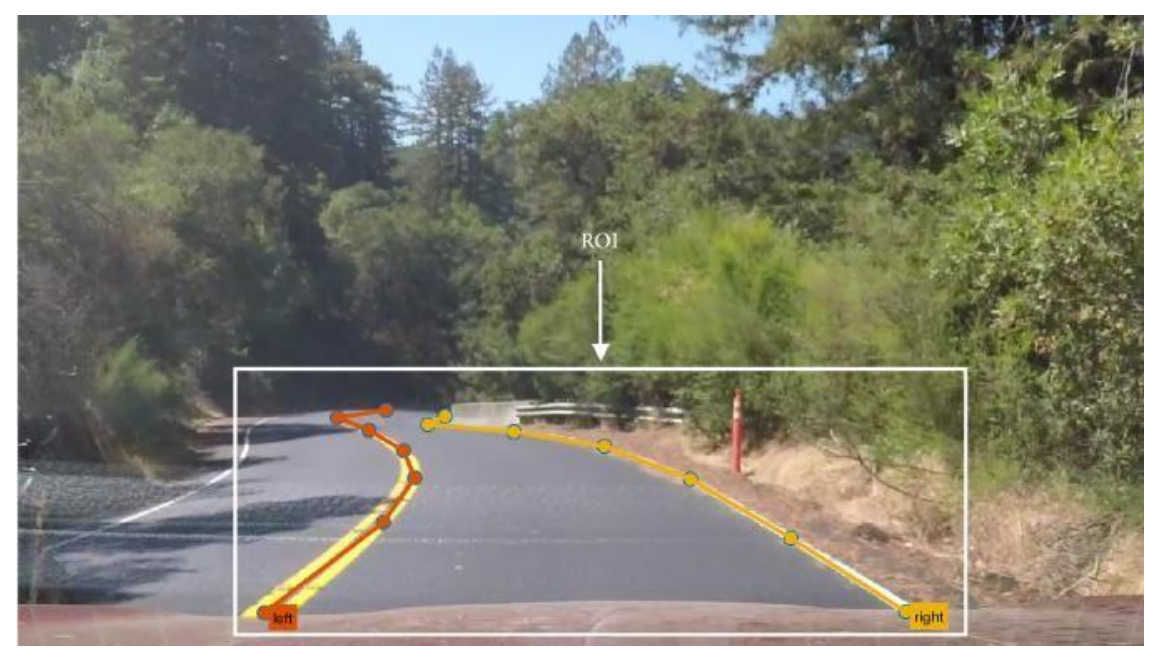

Figure:4 Initializing the Region of interest (ROI)

The original sequence of frames from video/computer vision are shown in figure-5 without detecting the lane boundaries in each frames.

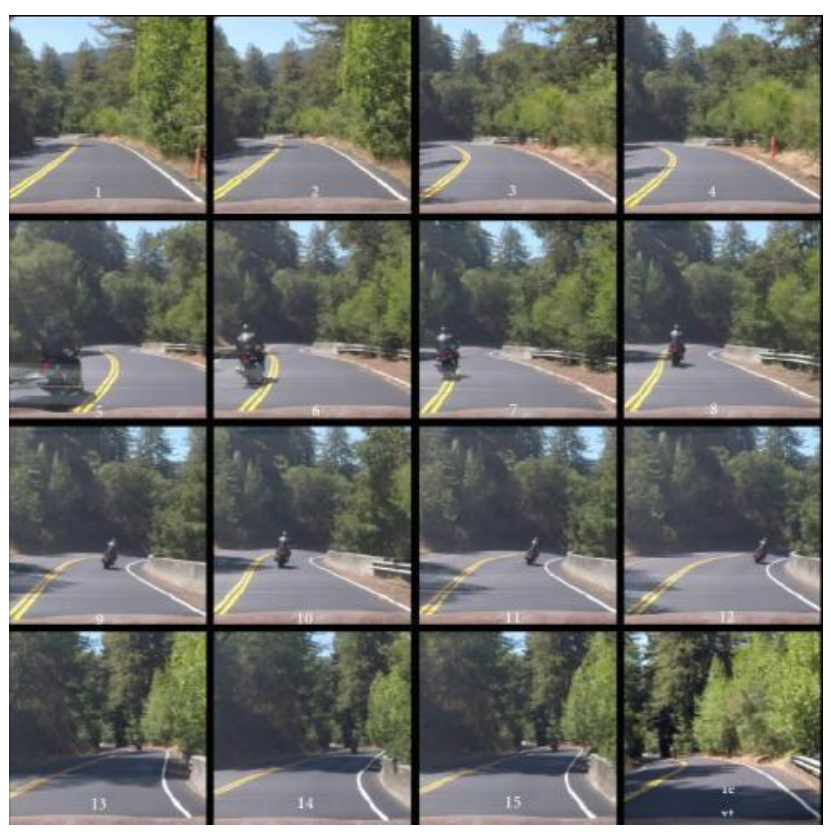

Figure:5 Original sequence of Frames from Video/computer vision 
Curve path / lane boundaries are detected in the frames by using the automated curve path detection algorithm as shown in the figure- 6 .

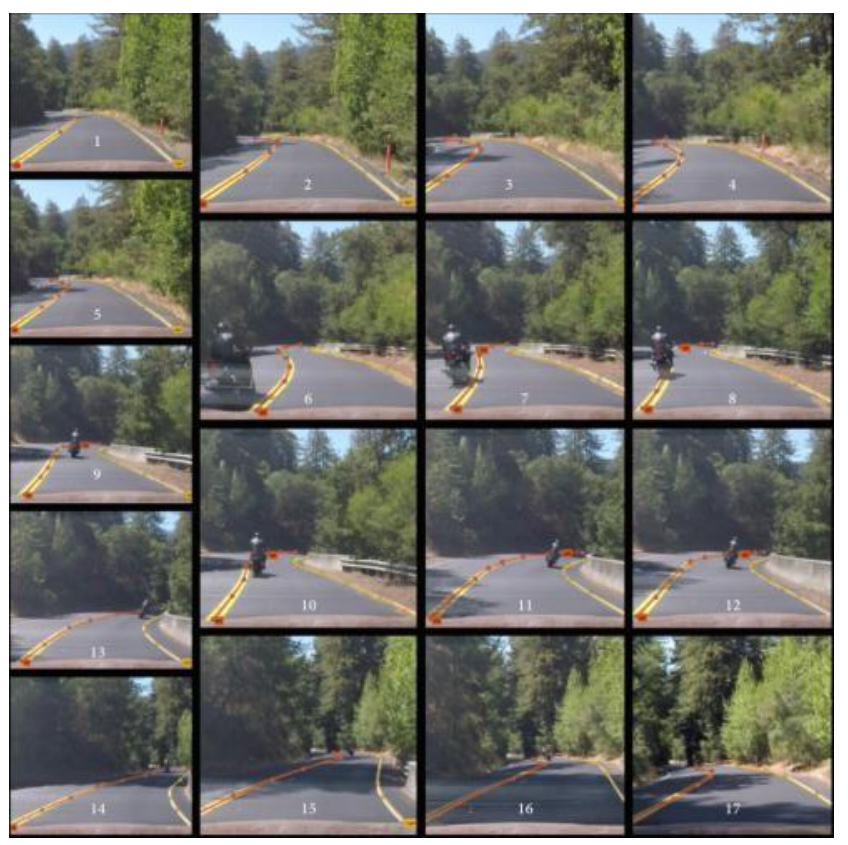

Figure:6 Frames after detecting the curve path using automated curve path detection algorithm

\subsection{Computation efficiency and accuracy:}

The computation efficiency and accuracy is depend upon the number of frames per given time, here the processing time is $0.04 \mathrm{sec}$ per frame by that we can say that 225 frames are computing per second. And the accuracy of the detection depends upon the no of frames processing per second. From the above results $80-90 \%$ accuracy is found by calculating from the equation- 1 as given below,

$$
\mathrm{C} \propto \mathrm{N}_{\mathrm{F}}
$$

Where $\mathrm{C}$ is the computation efficiency and $\mathrm{N}_{\mathrm{F}}$ is the number of frames per second are directly proportional to each other.

\section{CONCLUSION}

The curve path detection in autonomous vehicles play vital rule in field of self-driving cars and mobile robots. Mainly this technique is used in the hill areas where dead curves are present to increase the safety and avoiding the accidents the curve path detection in autonomous vehicles are necessary. This paper is focused mainly on the curve path detection using video/ computer vision in which deep learning technique is used to develop the lane/ curve path detection. To test the algorithm a real time video has been taken and executed the algorithm in the video and it is worked perfectly as shown in the results and discussion section.

\section{REFERENCES}

[1].Kodi Balasriram, Tanmay Preeven Pawar, Manimozhi M, "Terrain Classification in Automobiles using Artificial Neural Networks (ANN)” Research gate -2018.

[2].Haibo Jiang, Yilong Xiao, Yunwei Zhang, Xiaojing Wang, Haijiang Tai, "Curve path detection of unstructured roadsfor the outdoor robot navigation", Mathematical and computer modelling, 27 October 2011.

[3].Yue Wang, Eam Khwang Teoh, Dinggang Shen, "Lane detection and tracking using Bsnake", Image Vision Computing, 1 October 2003. 
[4].Zehang Sun, George Bebis, Ronald Miller, "On-Road Vehicle detection Using Optical Sensors: A Review", eTreppid Technologies, Computer Vision Laboratory, University of Nevada, Vehicle Design R\&A Department, Ford Motor Company.

[5].Nan Gao, Long Zhao, "An integrated land vehicle navigation system based on context awareness", Springer-Verlang Berlin Heidelberg 2015.

[6].Heba Aly, Anas Basalamah, Moustafa Youssef, "LaneQuest: An accurate and EnergyEfficient Lane detection system", SICE Annual Conference 2008, The University Electro-Communications, Japan, August 20-22,2008.

[7].D Zhang, K Li, J Wang, "Radar-based target identification and tracking on a curved road", The International Journal of Automobile Engineering, March 22015.

[8].Kevin McFall, "Using Visual Lane Detection to control steering in a self-driving vehicle", Department of Mechatronics Engineering, Kennesaw State University, 1100 South Marietta Parkway, marietta, GA,30060.

[9].Mark E. Russell, Arthur Crain, Anthoney Curran, Richard A. Campbell, Clifford A. Drubin, Willian F. Miccioli, "Millimeter-Wave Radar Sensor for Automotive Intelligent Cruise Control (ICC)", IEEE TRANSACTIONS ON MICROWAVE THEORY AND TECHNIQUES, Vol. 45, No. 12, December 1997.

[10]. Xiaodong Miao, Shunming Li, Huan Shen, "On-board lane detection system for intelligent vehicle based on monocular vision", International Journal on Smart Sensing and Intelligent systems, Dec 1,2012.

[11]. Alexey Voronov, Johan Hulten, Johan Wedlin, Cristofer englund, "Radar reflecting pavement markers for vehicle automation".

[12]. Joshue Perez Rastelli, Ray Lattarulo, Fawzi Nashashibi, "Dynamic Trajectory Generation Using Continuous-Curvature Algorithms for Door to Door Assistance Vehicles", IEEE Intelligent Vehicles Symposium, June 8-11, 2014, Dearborn, Michigan, USA.

[13]. Young Seop Son, wonhee Kim, Seung-Hi Lee, Chung Choo Chung, "Robust Multirate Control Scheme With Predictive Virtual Lanes for Lane-Keeping System of Autonomous Highway Driving", IEEE TRANSACTIONS ON VEHICULAR TECHNOLOGY, Vol-64, No-8, August 2015.

[14]. Byung-Hyun Lee, Sung-Hyuck Im, Moon-Beom Heo, Gyu-in Jee, "Curve Modeled Lane and stop line Detection Based GPS Error Estimation Filter", IEEE intelligent Vehicles Symposium, June 28- July 1, 2015, COEX, Seoul, Korea.

[15]. Ravi kumar satzoda, Mohan M. Trivedi, " On Performances Evaluation Metrics for Lane Estimation”, IEEE International Conference on Pattern Recognition, 2014.

[16]. Quoc-Bao Truong and Byung-Ryong Lee, "New Lane Detection for Autonomous Vehciles Using Computer Vision", International Conference on Control, Automation and Systems 2008, Oct. 14-17, 2008 in COEX, Seoul, Korea. 